\title{
Analysis of Stiffness in Extracellular Matrix Embedded with Bio-Conjugated Magnetic Beads in a Magnetic Field
}

\author{
Yue Du, Sahan C. B. Herath, Qing-Guo Wang, Harry Asada, and Peter C. Y. Chen
}

\begin{abstract}
In this paper we present a new model for determining the local stiffness of an extracellular matrix (ECM) sample embedded with bio-conjugated magnetic beads under the influence of an external magnetic field. In this model, the viscoelastic deformation of such ECM samples is analyzed using the finite element method. We report results from numerical simulations using our model on two typical scenarios for studying the pre-tension in the ECM caused by beads under a magnetic field. The analytical results are in close agreement with that obtained from COMSOL. We also applied our model on an actual ECM sample embedded with bio-conjugated beads and compared our analytical results with that obtained from stretch tests done on that sample. These results are comparable to that from the stretching tests.
\end{abstract}

Index Terms - Extracellular matrix, finite element analysis, pre-tension, stiffness, viscoelasticity

\section{INTRODUCTION}

Cell morphology and cytoskeletal structure are known to be influenced by the stiffness of their surrounding microenvironment (i.e., the extracellular matrix, or ECM) [1]-[3]. Cell migration has also been demonstrated to be significantly impacted by the ECM stiffness that determines the level of resistance to traction forces generated by cells. Various methods for modifying ECM stiffness have been used to explore its effect on cell behavior. These methods include changing the concentration [4],[5] or the $\mathrm{pH}$ value of the collagen [6],[7], or varying the boundary condition (e.g., floating gel or mechanically constrained gel) [2] or the polymerization condition of the ECM [8].

In an earlier paper we have proposed an approach for manipulating the local stiffness of ECM by embedding magnetic beads that are bio-conjugated with the ECM fibrils, then applying an external magnetic field to alter the fibrils' resistance to deformation [9]. In this approach, the local stiffness of an ECM sample can be manipulated in real-time by proper control of the external magnetic field. It has the potential to provide convenience and flexibility in generating stiffness gradients within a single ECM sample for studying cell behavior under varying ECM stiffness conditions.

Manuscript received August 7, 2012; revised September 16, 2012.

D. Yue, Sahan C. B. Herath, and Peter C. Y. Chen are with the Department of Mechanical Engineering, National University of Singapore, Singapore and Biosystem and Micromechanics Interdisciplinary Research Group, Singapore-MIT Alliance for Research and Technology Program, Singapore (e-mail: mpechenp@nus.edu.sg).

W. Q. Guo is with the Department of Electrical and Computer Engineering, National University of Singapore, Singapore.

H. Asada is with the Department of Mechanical Engineering, Massachusetts Institute of Technology, USA and Biosystem and Micromechanics Interdisciplinary Research Group, Singapore-MIT Alliance for Research and Technology Program, Singapore.
In this paper, we present a finite-element model for determining the local stiffness of an ECM sample embedded with bio-conjugated beads under the influence of an external magnetic field. Section II discusses the modification of ECM for manipulating the stiffness of ECM. Section III describes our finite-element method for analyzing viscoelastic deformation of ECM gel embedded with bio-conjugated beads, while Section IV discusses the calculation of magnetic force induced on the beads in the magnetic field generated by a permanent magnet. In Section $\mathrm{V}$ we apply this finite-element model to simulate the pre-tension generated in ECM by (i) a single bead, and (ii) two columns of aligned beads, and compare the results to that obtained using COMSOL. We also apply our finite-element model to an ECM sample with randomly distributed beads, and compare the analytical results (in terms of the percentage change in ECM stiffness) with experimental results obtained from a stretch test done on an actual ECM sample. Simulations are also conducted to reveal the influence of the size and concentration of beads on the change in ECM stiffness. We discuss possible improvements for this proposed model in Section VI.

\section{Modification of Extracellular Matrix}

Magnetic beads of radius about $1 \mu \mathrm{m}$ are embedded in collagen solution prior to the gelation of collagen. The streptavidin-coated beads are able to bind onto collagen fibrils during the gelation. Fig. 1 is one example of the confocal reflectance image of collagen fibrils and beads after the gelation. The bright dots are streptavidin-coated magnetic beads and the bright lines are Rat Tail Type I collagen fibrils Beads in very small size (the radius in about $1 \mu \mathrm{m}$ ) with a mediate concentration of less than $0.5 \mathrm{mg} / \mathrm{ml}$ are chosen such that they will not have a significant impact on the physical properties of the ECM. When exposed to a magnetic field, the beads generate in the ECM a pre-tension (i.e., pre-tensile stress), which interacts directly with external forces exerted on the ECM, thus altering its characteristics of deformation.

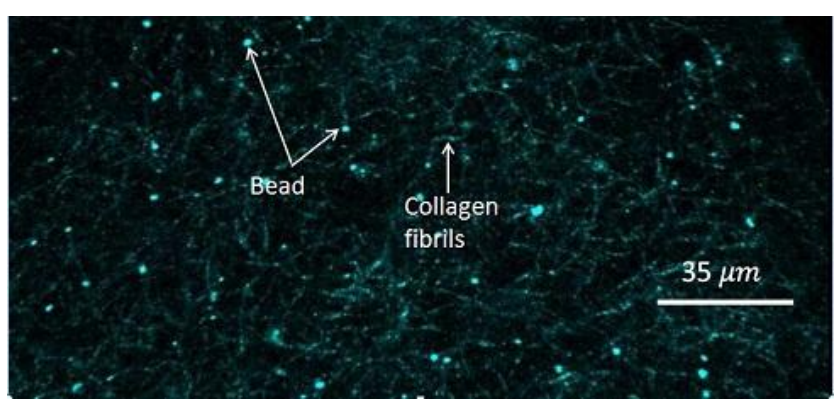

Fig. 1. Confocal reflectance image of collagen fibrils and beads. 


\section{FINITE ELEMENT ANALYSIS OF VISCOELASTIC DEFORMATION OF ECM EMBEDDED WITH BIO-CONJUGATED BEADS AND UNDER AN EXTERNAL MAGNETIC FIELD}

The modified ECM has a large amount of beads. This leads to a difficulty in constructing ECM model using the existing FEM tools as the beads are added in the ECM model one by one. Our model predicts viscoelastic deformation of the ECM under dispersed point loads imposed by the individual beads. The model extends the finite element method for elastic materials discussed in [10],[11]. The 8-node isoparametric solid finite element is used when meshing the ECM. The distribution of beads is assumed to be uniform inside the ECM. Thus the locations of the beads and the index of the elements containing the beads are known. We make another assumption that at most one bead is contained in an element, as the size of element is extremely small (about $0.002 \mathrm{~mm}$ $\times 0.01 \mathrm{~mm} \times 0.01 \mathrm{~mm})$.

The viscoelasticity of the ECM is represented by The Standard Linear Solid Model (SLSM) shown in Fig. 2. It consists of two branches in parallel: one having a spring and a dashpot in series and the other having only a spring. Compared to Maxwell and Kelvin-Voigt model, the deformation of viscoelastic materials reflected by the SLSM is more consistent with the actual situation. An instantaneous elastic deformation is observed when a viscoelastic material experiences an instantaneous stress. The existence of $E_{2}$ in the SLSM plays a role in generating such an instantaneous deformation. However, the full stress-strain relationship described by the SLSM is very complex. It is simplified in our case by limiting on constant stress. The source of the external force imposed on the ECM (which is already in pre-tension induced by the action of the external magnetic field on the beads) is usually considered to come from cells seeded on or inside it. Cell's traction force is assumed to be constant once the amount of cells and the mechanical properties of ECM are fixed [12]-[14].

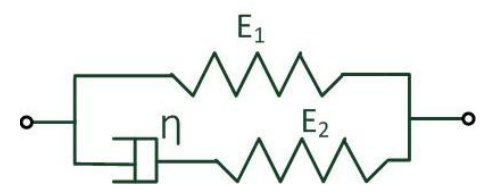

Fig.2. The standard linear solid model.

The stress-strain relationship under a constant stress can be expressed as

$$
\sigma_{e_{i n t}}=E_{1} \varepsilon_{e}+\eta \frac{E_{1}+E_{2}}{E_{2}} \dot{\varepsilon}_{e}
$$

where $\sigma_{e_{i n t}}$ and $\varepsilon_{e}$ are the stress and the strain for each element, respectively, $E_{1}$ is the stable elastic modulus after relaxation, $E_{2}$ is the instantaneous elastic deformation when a constant stress is applied, and $\eta$ is the viscosity of the dashpot.

Instead of combining three-dimensional deformation in one vector and calculating them simultaneously, we calculate the deformation in each dimension separately. Both methods generate the same result because they use the fixed relationship (i.e., the Poisson's ratio) to relate the deformation in one dimension to that in the other two. The following equations concern the deformation in one direction. The conversion of it to deformation in other two directions is demonstrated in the procedure to calculate three-dimensional deformations discussed later on in this section.
The strain can be obtained from the deformation by $\varepsilon_{e}=\partial u_{e} / \partial x$. This equation is usually written as

$$
\varepsilon_{e}=B_{e} u_{e}
$$

where $B_{e}$ is called as the shape function. Combining the relationship between stress and strain and the relationship between force and stress, we can obtain the force corresponding to the deformation for each element as

$$
\begin{gathered}
F_{e_{\text {int }}}=A_{e} E_{1} B_{e} u_{e}+A_{e} \eta \frac{E_{1}+E_{2}}{E_{2}} B_{e} \dot{u}_{e} \\
K_{e_{\text {elastic }}}=A_{e} E_{1} B_{e} \\
K_{e_{\text {viscous }}}=A_{e} \eta \frac{E_{1}+E_{2}}{E_{2}} B_{e}
\end{gathered}
$$

The element stiffness matrices $K_{e_{\text {elastic }}}$ and $K_{e_{\text {viscous }}}$ are then assembled to describe the whole ECM based on the shape function, with $A_{e}$ being the cross-sectional area of each element.

To formulate solid and structural mechanics problem, two groups of methods are commonly used, namely differential equation formulation methods and variation formulation methods. In this paper, force method belonging to the first group is applied. This method calculates the stress by using the equilibrium equations and the reduced compatibility equations obtained from substituting the relationship between the stress and the strain into the compatibility equations. The balance between the internal and external forces is considered, i.e.,

$$
F_{\text {int }}=F_{\text {ext }}
$$

$F_{\text {ext }}$ may include cell traction force, boundary forces and point loads exerted by magnetic field through beads. Then, the assembled external force at time step $t$ is equal to

$$
F_{\text {ext }}^{t}=K_{\text {elastic }} u^{t-1}-K_{\text {viscous }} \frac{\left(u^{t}-u^{t-1}\right)}{\Delta t}
$$

where $u^{t}$ is the global displacement of the nodes. Following the approach described in [15], the displacements of the nodes at the boundaries (usually equal to zeros) are assigned to them by small modifications of the stiffness matrices and the load matrices. Based on Equation (5), the displacement of each node at time step $t$ is determined recursively from its displacement at time step $(t-1)$, i.e.,

$$
u^{t}=\frac{\Delta t}{K_{\text {viscous }}^{-1}}\left(K_{\text {elastic }} u^{t-1}+\frac{K_{\text {viscous }}}{\Delta t} u^{t-1}-F_{\text {ext }}^{t}\right)
$$

The deformation in the $v$ and $w$ directions are derived from that in the $u$ direction at time step $t$ according to Poisson's effect.

Procedure to calculate three-dimensional deformation:

1) Initialization

a) Meshing ECM

b) Calculating the stiffness matrices $\mathrm{K}_{\mathrm{e}_{\text {elastic }}}$ and $\mathrm{K}_{\mathrm{e}_{\text {viscous }}}$ for each element and assembling them

c) Calculating magnetic force exerted on beads (point loads on ECM) using the method discussed in Section IV

d) Searching on elements containing beads and allocating point load to them

e) Assigning the initial displacement of nodes

2) For each time step $t$ 
a) Disassembling the external force into $\mathrm{F}_{\mathrm{e}_{\mathrm{ext}}}$ in terms of elements

b) Combining different types of load matrices

c) Calculating $\mathrm{K}_{\text {elastic }} \mathrm{u}^{\mathrm{t}-1}$ and $\mathrm{K}_{\text {viscous }} \mathrm{u}^{\mathrm{t}-1} / \Delta \mathrm{t}$, where $\Delta \mathrm{t}$ is the time interval and $\mathrm{u}^{\mathrm{t}-1}$ is the nodes displacement vector at the last time step

d) Calculating $\mathrm{u}^{\mathrm{t}}$ based on Equation (6)

e) Calculating $v^{t}$ and $w^{t}$ according to the relationship with $\mathrm{u}^{\mathrm{t}}$ in terms of Poisson's ratio

End for

\section{CAlculation of Magnetic ForCE}

We adopt the method developed in [16] and [17] to calculate the force experienced by a bead exposed to a magnetic field. The magnetic poles of the permanent magnet are considered as a current loop when calculating the magnetic field using the Biot-Savart law. The magnetic field is subsequently applied in Equation (7) shown below to calculate the magnetic force.

$$
F=\left\{\begin{array}{lr}
\frac{1}{2} \nabla(\vec{m} \cdot \vec{B}) & \vec{m}(\vec{B}) \text { saturates } \\
\frac{V \chi_{\text {mass }} \nabla|\vec{B}|^{2}}{2 \mu_{0}} & \text { otherwise }
\end{array}\right.
$$

Fig. 3 plots the magnetic force experienced by a bead over a range of distance between the bead and the magnet. Each bead (BM551 from Bangs Laboratory, Inc.) has a radius of about $1 \mu \mathrm{m}$ with a saturation magnetization of about 70 $\mathrm{emu} / \mathrm{g}$. The cubic permanent magnet with a dimension of $12 \mathrm{~mm} \times 12 \mathrm{~mm} \times 4 \mathrm{~mm}$ and a remnant magnetic field equal to $1.5 \mathrm{~T}$ is used. It can be seen from Fig. 3 that the magnetic force induced on the bead decreases with an increase in the distance from magnet, and the magnitude of this force increases with the bead radius. A force of about $0.45 \mathrm{nN}$ can be reached for a bead with a radius of $1.5 \mu \mathrm{m}$ located within 1 $\mathrm{mm}$ from the magnet.

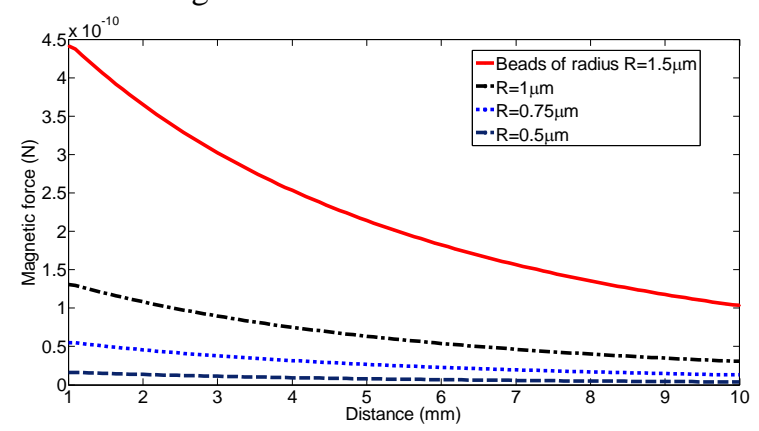

Fig. 3. The magnetic force over distance on beads of different sizes.

\section{NUMERICAL SimULATION AND VALIDATION OF RESUlts}

\section{A. Calculation of Pre-Tension in Ecm Caused by Beads Using The Proposed Model and Comsol}

The pre-tension caused by the beads exposed to a magnetic field is simulated using both the derived finite-element model and COMSOL. Construction of an ECM model using COMSOL is restrictive due to the limit on the number of beads that can be included in the model. For this reason, we designed two small-scale (in terms of the number of beads involved) scenarios. The first concerns an ECM with only one embedded bead, while the second scenario has the arrangement of two columns of beads in the ECM under opposite magnetic forces, as is illustrated in Fig. 4a) and Fig. 4b).

The viscoelastic parameters used are $E_{1}=1719 \mathrm{~Pa}, E_{2}=$ $500 \mathrm{~Pa}, \eta=137 \mathrm{~Pa} \cdot \min$. For the case of a single bead, the bead is set at $0.003 \mathrm{~m}$ away from one edge of the ECM. The opposite edge is fixed. The force exerted by the bead due to an external magnetic field is set at $1.2 \times 10^{-7} \mathrm{~N}$. In this scenario, the pre-tension in the area near the bead in the direction of the bead-exerted force is analyzed, as illustrated in Fig. 4a).

For the beads-in-two-column scenario, each column is set at a distance of $0.003 \mathrm{~m}$ away from an edge of an ECM strip with the magnetic forces acting in opposite directions as illustrated in Fig. 4b). The effect of the pre-tension due to a single bead is sensitive to the mesh fineness around the bead. For comparison with COMSOL, in the calculation using our model the size of a mesh element is similar to that used in the COMSOL simulation. This issue is not significant for the beads-in-two-column scenario, in which the pre-tension of the ECM in the direction perpendicular to the direction of the bead-exerted force is analyzed.
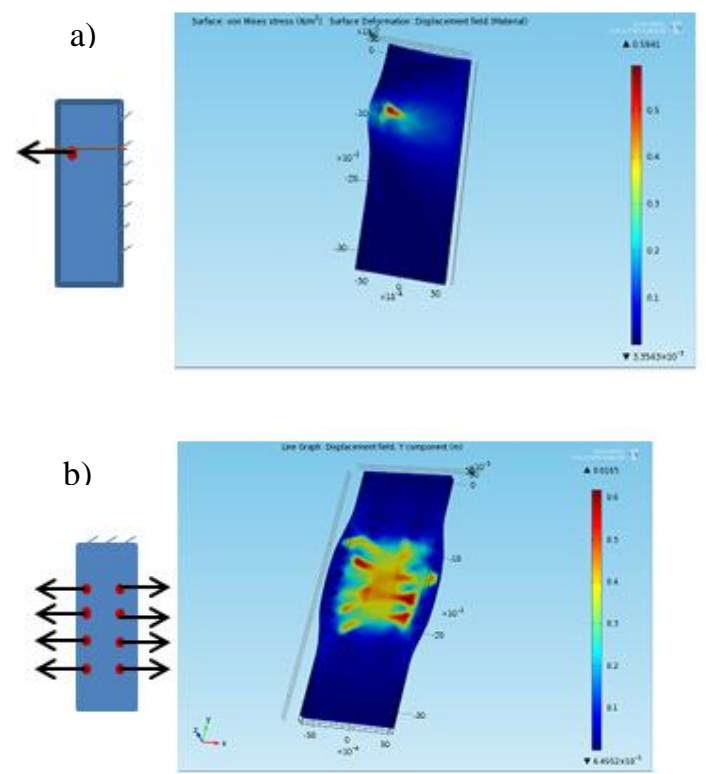

c)

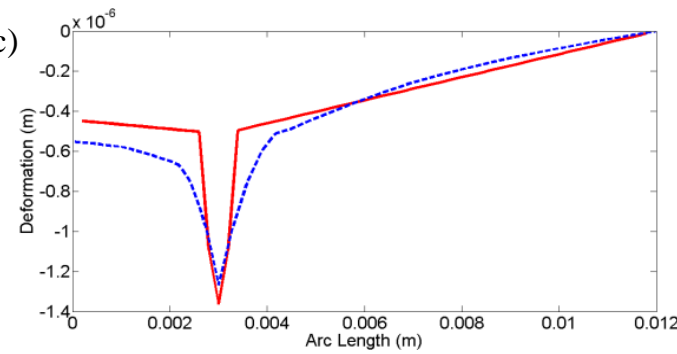

d)

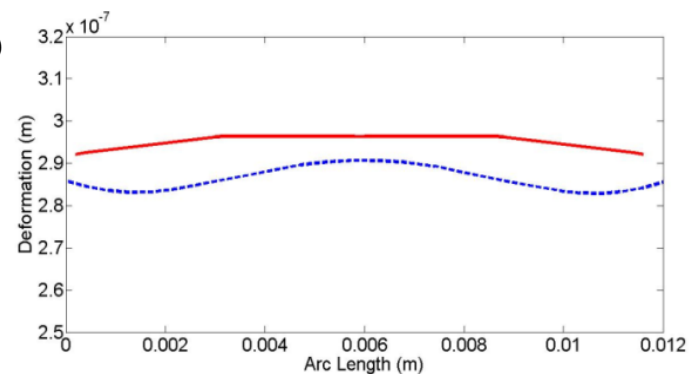

Fig. 4. Pre-tension created in ECM by point load. 
Fig. 4a) and Fig. 4b) show the results obtained from COMSOL for the stress tensor and the deformation at $\mathrm{t}=26 \mathrm{~s}$ for the two scenarios. Fig. 4c) and Fig. 4d) show the exact pre-tension generated in the ECM. The red and solid curve shows the results from our model and the blue and dashed curve shows the results from COMSOL. Fig. 4c) shows the pre-tension in the area near the bead in the direction of the bead-exerted force.

It can be seen that results from our finite-element model are comparable to that obtained from COMSOL. The maximal pre-tension at $t=26 \mathrm{~s}$ calculated from our model is $-1.4 \times 10^{-6} \mathrm{~m}$, while that from COMSOL is $-1.2 \times 10^{-6} \mathrm{~m}$ A sharp pre-tension is generated in a very small area near the bead, and decays away from it. Fig. 4d) shows the pre-tension of the ECM in the direction perpendicular to the direction of the bead-exerted force. The deformation calculated from our model is between $2.9 \times 10^{-7} \mathrm{~m}$ and $3 \times 10^{-7} \mathrm{~m}$, while that from COMSOL is between $2.8 \times 10^{-7} \mathrm{~m}$ and $2.9 \times 10^{-7}$.

\section{B. Change in Stiffness of Actual Ecm Sample with Bio-Conjugated Magnetic Beads Under Magnetic Field}

In this section we discuss the application of our model to predict the change in the measured stiffness of an ECM sample due to pre-tension generated by the bead-exerted force, and compare our analytical prediction with the actual results obtained from a stretch-test experiment conducted using an actual ECM sample, as reported in [9].

The setup of stretch-test experiment is shown in Fig. 5. The stretch test involved an ECM strip embedded with beads bio-conjugation with the collagen fibers. The strip was placed between two permanent magnets, with the bottom of the strip fixed and part of the strip in the middle directly exposed width-wise to the magnetic field. When the top of the strip is pulled, the beads in the mid-section of the strip exposed to the magnetic field experience a force which can be resolved into a horizontal component pointing to the magnets and a vertical component pointing downward. The downward force on a bead provides resistance to the deformation of the strip.

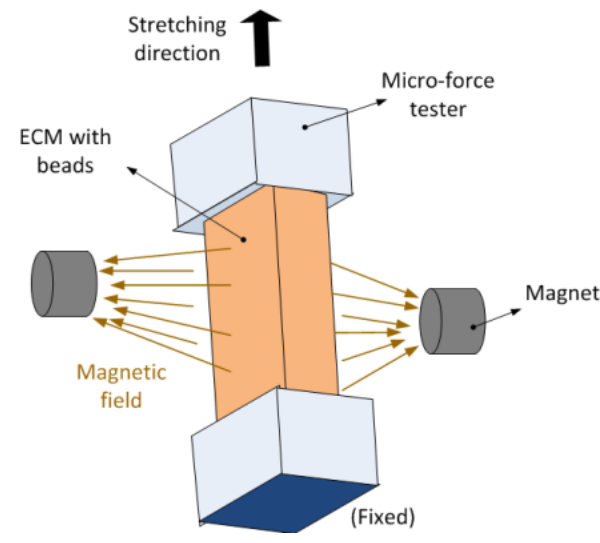

Fig. 5. The setup of the stretching test experiment.

Since in this case the beads are distributed randomly in the ECM strip, it is not practical to locate the exact position of each bead so that COMSOL can be used to study the resulting ECM stiffness (even if the number of beads under consideration is small). Our analytical model, however, can still be readily applied. The same parameter values used in the experiment (as summarized in Table I) are also used in this simulation. Using the value for the viscoelasticity of pure
ECM gel experimentally determined in [9], we obtain $E_{1}=1719 \mathrm{~Pa}$ and $\eta=137 \mathrm{~Pa} \cdot \min$ from our model.

TABLE I: PARAMETERS USED IN THE STRETCHING TEST EXPERIMENT

\begin{tabular}{lll}
\hline \hline Parameter & Value & Units \\
\hline The dimension of ECM & $33 \times 12 \times 4$ & $\mathrm{~mm}$ \\
Magnetic flux field & 1.5 & $\mathrm{~T}$ \\
Volume of beads sample & 0.315 & $\mathrm{ml}$ \\
Deformation rate of ECM & 10 & $\mathrm{~mm} / \mathrm{min}$ \\
Diameter of magnet & 12 & $\mathrm{~mm}$ \\
Thickness of magnet & 4 & $\mathrm{~mm}$ \\
Magnetization & 70 & $\mathrm{emu} / \mathrm{g}$ \\
\hline \hline
\end{tabular}

Fig. 6 shows the effect of beads concentration on the pre-tension generated by beads in the ECM and thus the stretching force that is required to deform the ECM at the set rate. Fig. 6a) plots the pre-tension (that acts against the stretching force) generated by beads of $1 \mu \mathrm{m}$ radius at a concentration of $0.02 \mathrm{mg} / \mathrm{ml}, 0.1 \mathrm{mg} / \mathrm{ml}$ and $0.5 \mathrm{mg} / \mathrm{ml}$ in the ECM sample in the presence of magnetic field within 16s. It can be seen that the pre-tension increases exponentially and finally approaches a stable value because of the viscoelasticity of the ECM gel. Moreover, the rate of increase in pre-tension is the highest for the ECM sample with a bead concentration of $0.5 \mathrm{mg} / \mathrm{ml}$.

Fig. 6b) shows the tensile forces needed to deform the ECM gel at a constant rate of $10 \mathrm{~mm} / \mathrm{min}$ in the absence and the presence of the magnetic field for different bead concentrations. It can be seen that the greater force is needed to deform the ECM in the presence of the magnetic field. Moreover, in the presence of magnetic field the increase in bead concentration results in a greater force required to deform the ECM. The reason is that the increase in bead concentration causes the increase in pre-tension that acts against the stretching force. There is an offset of $0.002 \mathrm{~N}$ for all the force curves corresponding to the portion of force that is needed to deform the viscous part of the ECM gel at a constant rate.

a)

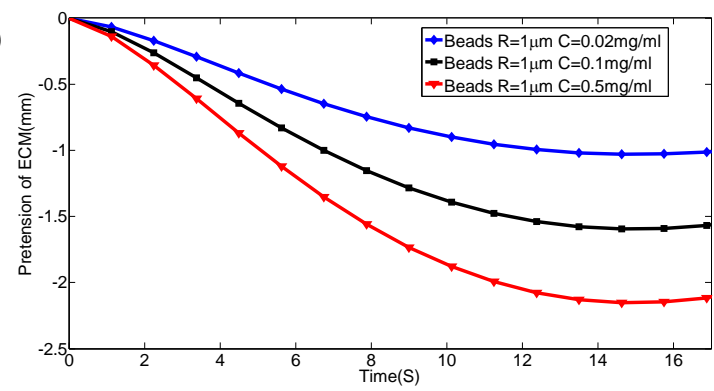

b)

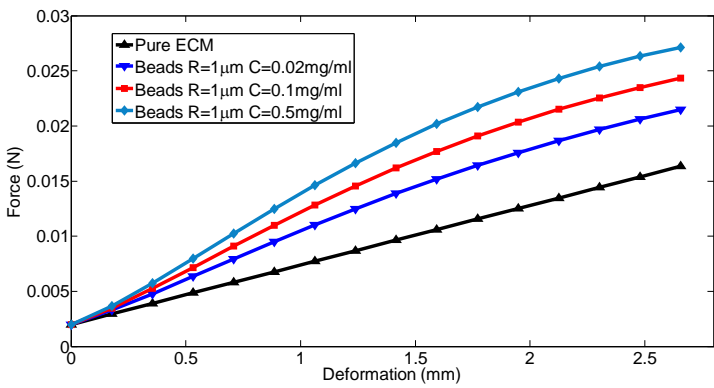

Fig. 6. The effect of beads concentration on the pre-tension in ECM generated by beads and the stretching force. 
Fig. 7 shows the effect of bead size on the formation of pre-tension in the ECM and on the stretching force to deform the ECM at the set rate. Fig. 7a) shows the pre-tension formed by beads of $0.75,1$ and $1.5 \mu \mathrm{m}$ radius with the same concentration of $0.1 \mathrm{mg} / \mathrm{ml}$ in a magnetic field for a period of $16 \mathrm{~s}$. It can be seen that greater pre-tension was generated by beads of larger size. The reason is that a large magnetic force can be induced on large bead compared to that on small ones. It can also be found that there is a significant increase in the pre-tension for beads of $1.5 \mu \mathrm{m}$ radius compared to that of 1 $\mu \mathrm{m}$ radius. Fig. $7 \mathrm{~b}$ ) shows the tensile forces needed to deform the ECM gel at a constant rate in the absence/presence of the magnetic field and in different beads size. It can be seen that a greater tensile force is needed to deform the ECM with beads in larger size. This is consistent with the trend of pre-tension for different sizes of beads.

a)

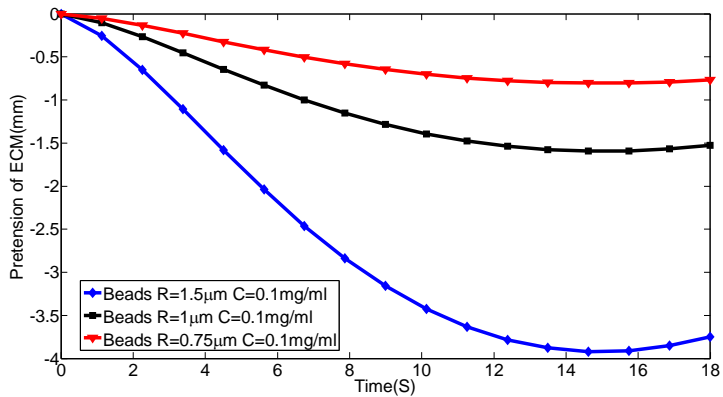

b)

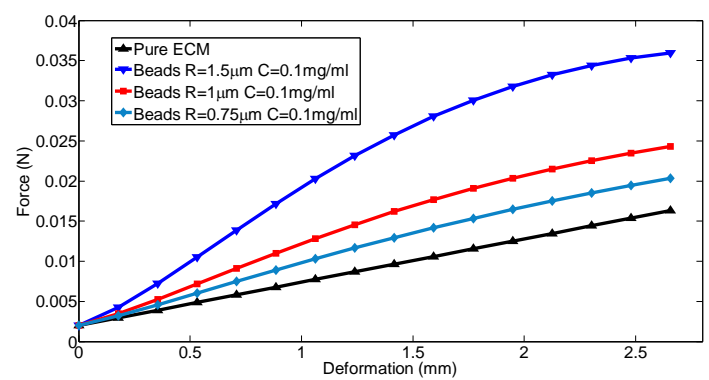

Fig. 7. The effect of beads size on the pre-tension in ECM generated by beads and the stretching force.

The apparent stiffness of the ECM can be calculated on the basis of the deforming force and the resulted deformation shown in Fig. 6b) for beads in different concentration and in Fig. 7b) for beads with different sizes. This is done by linearizing the curves and then measuring the slope of the linearized force-verse-deformation line. The stiffness was estimated at $t=16 \mathrm{~s}$ (shown in Fig. 8), at which point the pre-tension was at the stable value. The results of slope for two groups are shown. Group 1 consists of the values generated from Fig. $6 b$ while Group 2 from Fig. 7b).

It can be seen from Group 1 that the stiffness is increased by increasing beads concentration, while Group2 shows that large beads lead to a stiffer ECM due to the creation of greater pre-tension compared. Moreover, the change in the ECM apparent stiffness is more sensitive to the variation in bead size than to the variation in bead concentration.

Fig. 9 shows the percentage change in stiffness calculated using our model and that determined experimentally as reported in [9] for two types of samples with bead concentrations of $0.5 \mathrm{mg} / \mathrm{ml}$ and $0.1 \mathrm{mg} / \mathrm{ml}$. It can be seen that our analytical prediction of the change in ECM stiffness is in close agreement with the experimental results, especially for the case where the sample contained a higher concentration of beads.

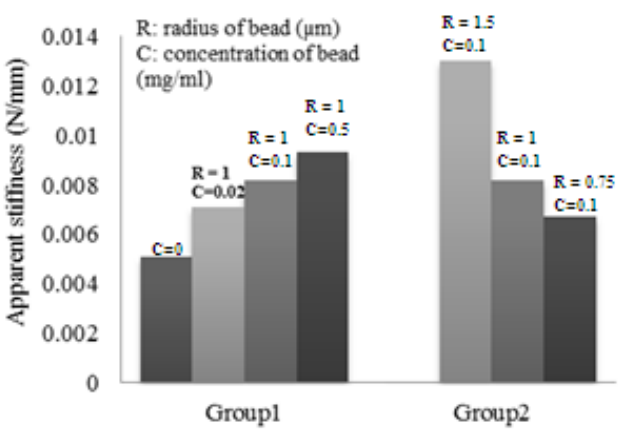

Fig. 8. Apparent stiffness of ECM with beads.

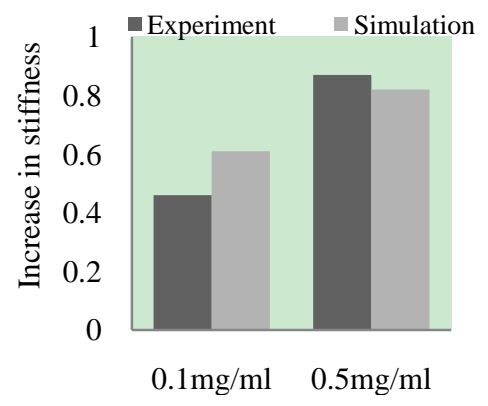

Fig. 9. Comparison of increase in stiffness.

\section{DISCUSSION AND CONCLUSION}

The viscoelastic deformation of ECM samples embedded with bio-conjugated magnetic beads in the presence and absence of an external magnetic field has been analyzed by a new model constructed based on the finite-element method. The role of each single bead is considered as exerting a point load on the ECM. The point load is equal to the magnetic force induced on the bead by the external magnetic field, and increases with the size of the bead. Based on information about viscoelastic deformation and external force, the stiffness of the ECM can be computed for the cases when the magnetic field is absent and present. We conducted numerical simulation using our model on two typical scenarios for calculating the pre-tension caused by beads and compared our simulation results with that obtained from COMSOL. The results from our model are in close agreement with that obtained from COMSOL. For bead of 1 $\mu \mathrm{m}$ radius and a force with a magnitude of about $0.1 \mu \mathrm{N}$, the maximum deformation of the ECM is about several microns and occurs near the bead (Young's module of ECM is about $1719 \mathrm{~Pa}$ and viscosity is about $137 \mathrm{~Pa} \cdot \mathrm{min}$ ).

We also applied our model on an actual ECM sample embedded with bio-conjugated magnetic beads. Simulation has been conducted to reveal the effect of bead concentration and size variation on the ECM stiffness. Specifically, the studies have been conducted on investigating the change in the formation of pre-tension in the ECM and the stretching force required to deform ECM at a prescribed constant rate. The pre-tension created by the beads in the ECM plays a significant role in resisting the applied stretching force. The results show that higher level of pre-tension and its rate of increase are associated with higher concentration of beads. Therefore, a greater force is needed to deform an ECM with 
higher bead concentration.

The work report in this paper contributes to the mathematical analysis of the change in ECM stiffness due to the creation of pre-tension in the ECM using micro-size magnetic beads under the influence of an external magnetic field. These results provide a analytical basis for developing sophisticated techniques for the control of the stiffness of ECM gel. Compared to other methods of manipulating ECM stiffness by changing the chemical composition of collagen gel, the method described in this paper is more versatile because it enables the control of the desired local stiffness at a particular region (with the presence and absence of magnetic beads) of an ECM sample and at a specific time (by switching on or off the external magnetic field). Such control will be useful in studying cell behavior in a microenvironment with varying stiffness.

The analysis presented in this paper has not addressed the issue of how the mere existence of the beads in the ECM may influence the stiffness of the ECM in the absence of the magnetic field. This issue concerns the physical properties of the beads and the ECM, and the bio-conjugating reaction between the beads and ECM. Taking this issue into account in the analysis will enable the resulting model to predict more accurately how the ECM will deform in the presence of an external magnetic field. Another issue that needs to be explored is the inclusion of cell traction forces in the model of ECM deformation, since such forces may interfere with the magnetic forces that are supposed to create the desired pre-tension in the ECM. The precision control of ECM stiffness by controlling the pre-tension providing certain level of resisting to external force is another interesting problem to be dealt with in subsequent research.

\section{ACKNOWLEDGMENT}

The authors would like to thank the BioSystem and Micromechanics Interdisciplinary Research Group (under the Singapore-MIT Alliance for Research and Technology Program) for their financial support. The first author also would like to acknowledge the financial support provided by The China Scholarship Council and the National University of Singapore, and the second author would like to acknowledge the financial support provided by the National University of Singapore.

\section{REFERENCES}

[1] S. Huang, C. S. Chen, and D. E. Ingber, "Control of cyclin D1, p27Kip1, and cell cycle progression in human capillary endothelial cells by cell shape and cytoskeletal tension," Mol. Biol. Cell, vol. 9, no. 11, pp. 3179-3193, Nov. 1998

[2] A. L. Sieminski, R. P. Hebbel, and K. J. Gooch, "The relative magnitude of endothelial force generation and matrix stiffness modulate capillary morphogenesis," Exp. Cell Res, vol. 297, no. 2, pp. 574-584, July 2004.

[3] S. Weng and J. Fu, "Synergistic regulation of cell function by matrix rigidity and adhesive pattern," Biomaterials, vol. 32 , no. 36 , pp. 9584-9593, Dec. 2011.

[4] S. Kanzawa, H. Endo, and N. Shioya, "Improved in vitro angiogenesis model by collagen density reduction and the use of type III collagen," Ann. Plast. Surg, vol. 30, no. 3, pp. 244-251, Mar 1993.

[5] A. Stéphanou, G. Meskaoui, B. Vailhé, and P. Tracqui, "The rigidity in fibrin gels as a contributing factor to the dynamics of in vitro vascular cord formation," Microvasc. Res, vol. 73, no. 3, pp. 182-190, May 2007.

[6] N. Yamamura, R. Sudo, M. Ikeda, and K. Tanishita, "Effects of the mechanical properties of collagen gel on the in vitro formation of microvessel networks by endothelial cells," Tissue Engin, vol. 13, no. 7, pp. 1443-1453, July 2007.

[7] V. Nehls and R. Herrmann, "The configuration of fibrin clots determines capillary morphogenesis and endothelial cell migration," Microvasc. Res, vol. 51, no. 3, pp. 347-364, May 1996.

[8] C. F. Deroanne, C. M. Lapiere, and B. V. Nusgens, "In vitro tubulogenesis of endothelial cells by relaxation of the coupling extracellular matrix-cytoskeleton," Cardiovasc. Res, vol. 49, no. 3, pp 647-658, Feb 2001.

[9] C. Y. Chen Peter, S. C. Herath, Dong-An Wang, K. Su, K. Liao, and H. Asada, "Active manipulation of uniaxial ECM stiffness by magnetic anchoring of bio-conjugated beads," in Proc. ASME Summer Bioengin. Conf. Pennsylvania, 2011, pp. 1-14.

[10] T. R. Chandrupatla and A. D. Belegundu, Introduction to Finite Elements in Engineering, 2nd ed. Harlow, New Jersey: Prentice Hall, 1996, ch. 3, pp. 46-85

[11] G. Dhatt and G. Touzot, The Finite Element Method Displayed, Chichester, U. K.: John Wiley and Sons, 1984, ch4.

[12] D. Manoussaki, S. R. Lubkin, R. B. Vernon, and J. D. Murray, "A mechanical model for the formation of vascular networks in vitro," Acta Biotheor, vol. 44, pp. 271-282, Nov. 1996.

[13] N. V. Mantzaris, S. Webb, and H. G Othmer, "Mathematical modeling of tumor-induced angiogenesis," J Math Biol, vol. 49, pp. 111-187, Feb 2004.

[14] M. J. Holmes and B. D. Sleeman, "Mathematical model of tumour angiogenesis incorporating cellular traction and viscoelastic effects," $J$ Theor Biol, vol. 202, no. 2, pp. 95-112, Jan. 2000.

[15] S. S. Rao, The Finite Element Method in Engineering, $4^{\text {th }}$ ed. Burlington, MA: Elsevier ButterWorth-Heinemann, 2005, ch. 8, pp 277-306.

[16] J. Lipfert, X. M. Hao, and N. H. Dekker, "Quantitative modelling and optimization of magnetic tweezers," Biophys $J$, vol. 96, no. 12, pp.5040-5049, June 2009.

[17] P. Kollmannsberger and B. Fabry, "High-force magnetic tweezers with force feedback for biological applications," Rev Sci Instrum, vol. 78, no.11, pp. 114301, Nov 2007.

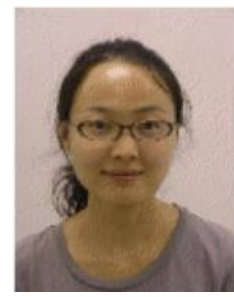

Yue Du received the B.S. degree from Department of Mechanical Engineering and Automation, Northeast Agricultural University, Harbin, China, in 2007, and the M.S. degree from Department of Mechatronics, Harbin Institute of Technology, Harbin, China, in 2009. She is currently pursuing a Ph.D. degree at the Department of Mechanical Engineering, National University of Singapore, Singapore. Her research interests include instruments and sensors, control, image processing and biomechanics.

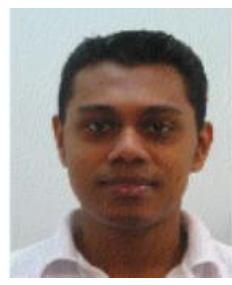

Sahan C. B. Herath received the B.S. degree from Department of Mechanical Engineering, National University of Singapore, Singapore, in 2009. He is currently pursuing a Ph.D. degree at the Department of Mechanical Engineering, National University of Singapore, Singapore. His research interests include electromechanical systems, design and control.

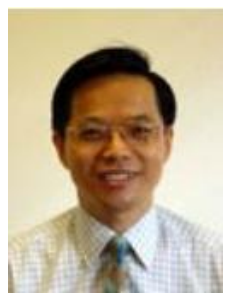

Qing. Guo Wang received the B.S., M.S. and Ph.D. degree in 1982, 1984 and 1987, respectively, all from the Zhejiang University, Zhejiang, China.Since 1993 $\mathrm{He}$ has been with the National University of Singapore, Singapore, where he is currently a Professor with the Department of Electrical and Computer Engineering. His research interests are in modeling, estimation, prediction, control, optimization and automation for complex systems. 


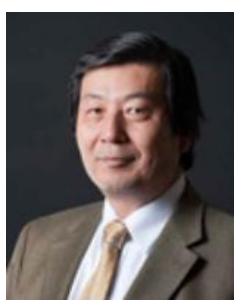

H. Harry Asada received the B.S. degree in Mechanical Engineering, and M.S. and Ph.D. degrees in Precision Engineering in 1973, 1975, and 1979, respectively, all from Kyoto University, Japan. $\mathrm{He}$ joined the Massachusetts Institute of Technology faculty in 1982, where he is currently the Ford Professor of Engineering and Director of the Brit and Alex d'Arbeloff Laboratory for Information Systems and Technology in the Department of Mechanical Engineering at the M.I.T. His research focuses on the area of robotics, bioengineering, and dynamic systems and control.

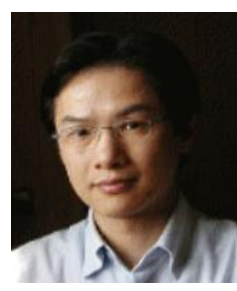

Peter C. Y. Chen received the Ph.D. degree from the University of Toronto, Canada, in 1995. He joined the National University of Singapore (NUS) in April 2000, where he is currently an Associate Professor with the Department of Mechanical Engineering. Prior to joining NUS, he worked on various projects for China International Marine Container Ltd., Automation Tooling System, Inc., General Dynamics Corp. (Electronics Division, USA), Philips Pte. Ltd., the University of Toronto, CRS Robotics Inc., the Canadian Space Agency, and the Singapore Institute of Manufacturing Systems. His research interests center on mechanical manipulation of biological systems, and supervisory control of discrete-event systems. 